\title{
LA CAIÍDA DE “CENIZA” PROVENIENTE DEL VOLCAN IRAZÚ (COSTA RICA) EL 8 DE DICIEMBRE DE 1994: ¿UNA EXPLOSIÓN FREÁTICA?
}

\author{
THE “ASHFALL” COMING FROM IRAZÚ VOLCANO (COSTA RICA) ON \\ DECEMBER 8 ${ }^{\mathrm{TH}}$ 1994: A PHREATIC EXPLOSION?
}

\author{
Guillermo E. Alvarado ${ }^{1,2 *}$, Mauricio M. Mora $^{3} \&$ Andrés Ulloa $^{1}$ \\ ${ }^{1}$ Centro de Investigaciones Geológicas, Universidad de Costa Rica \\ ${ }^{2}$ Área de Amenazas y Auscultación Sísmica y Volcánica, ICE, \\ Apdo. 10032-1000, Costa Rica \\ ${ }^{3}$ Escuela Centroamericana de Geología, Universidad de Costa Rica \\ *Autor para contacto: galvaradoi@ice.go.cr
}

(Recibido: 12/2/2013; aceptado: 11/6/2013)

\begin{abstract}
During the night of December $8^{\text {th }}$, 1994, along an unstable area at the northern flank of Irazú volcano, Costa Rica, a small volcanic debris avalanche and subsequent debris flows took place, followed by an "ashfall" that reached $30 \mathrm{~km}$ southwest from the volcano. At that time, these processes were interpreted as the result of a phreatic explosion. However, a review of the descriptions given in the technical reports and other documents of the time, together with the analysis of the geomorphology obtained from aerial photographs taken on December 20, 1994, by the Costa Rica National Geographic Institute, lead to discuss the validity of this interpretation, which although not ruled out, it seems unlikely. This paper proposes, based on the facts and information available, a new hypothesis of the occurrence of a volcanic debris avalanche resulting from a major landslide near the northern summit area. Consequently, the following "ash" fall could be interpreted as generated by the dust produced byauto-fragmentation of the debris avalanche, so subsequently called co-debris avalanche dust-cloud deposit.
\end{abstract}

Keywords: Phreatic explosion, debris avalanche, co-debris avalanche dust-cloud, debris flow, Irazú, Costa Rica

RESUMEN: Durante la noche del 8 de diciembre de 1994, en un área inestable del flanco norte del volcán Irazú, ocurrió una avalancha de escombros volcánicos con lahares asociados, así como la caída de "ceniza" que alcanzó hasta unos $30 \mathrm{~km}$ al suroeste del volcán. En ese entonces, los procesos, fueron interpretados como el resultado de una explosión freática. Sin embargo, la revisión de las descripciones expuestas en los informes científicos y otros documentos de la época, en conjunto con el análisis de la geomorfología obtenida a partir de fotografías aéreas tomadas el 20 de diciembre de 1994, por el Instituto Geográfico Nacional, nos conduce a discutir sobre la validez de esa interpretación, que aunque no se descarta, parece improbable. En este trabajo se propone que los hechos e información disponible nos acercan más a la hipótesis de la ocurrencia de la avalancha de escombros volcánicos producto de un deslizamiento de un segmento septentrional importante cercano a la cúspide y, en consecuencia, la "ceniza" podría ser interpretada como el polvo producto de la auto-fragmentación de los bloques rocosos de la avalancha.

Palabras clave: Explosión freática, nube de polvo acompañante de avalancha de escombros volcánicos, lahar, Irazú, Costa Rica 


\section{INTRODUCCIÓN}

El sector septentrional del Irazú es conocido desde hace décadas como un sector donde suelen ocurrir grandes deslizamientos y por la presencia de fumarolas y fuentes termales. Se sabe que la actividad fumarólica se ha mantenido al menos desde el siglo antepasado con temperaturas entre $85-95^{\circ} \mathrm{C}$, las cuales son controladas por la condensación del vapor de agua (Alvarado, 2011, y referencias allí citadas). También Alvarado (1987, 1993) había caracterizado esta zona como de potencial alto para la generación de deslizamientos y la formación de un nuevo cráter.

Lo sucedido en el flanco norte el 8 de diciembre de 1994, a las 10:48 p.m. (hora local), quedó plasmado en la historia del volcán Irazú como el resultado de una o varias explosiones freáticas, que a su vez generaron deslizamientos, corrientes de barro y la caída de ceniza hacia el oeste del volcán, según las descripciones e interpretaciones vertidas en documentos científicos de la época, tanto a nivel nacional (Barquero et al. 1995a; Fernández et al., 1994; GVN, 1994, 1995; RSN, 1994, 1995; Mora et al., 1996; Mora, 1997; Barquero, 1998) como internacional (Siebert et al., 2010).

Este trabajo tiene como objetivo situar los acontecimientos del 8 de diciembre de 1994 en el volcán Irazú (Fig. 1), en el contexto del conocimiento volcanológico actual, a partir de:

1. La revisión de la literatura y fotografías de la época.

2. El análisis geomorfológico de las fotografías aéreas del Instituto Geográfico Nacional de 1978 a escala 1: 35000 y del 20 de diciembre de 1994 a escala 1: 10000 (número 133-135).

3. La comparación con la avalancha ocurrida en Arancibia (Cordillera de Tilarán) en el año 2000.

\section{ANTECEDENTES}

Antes del año 1991, la sismicidad en el volcán Irazú era muy baja. Sin embargo, a partir de enero de ese año se incrementó y permaneció fluctuante hasta disminuir casi por completo en abril de 1992. A partir de mayo de 1991, también aparecieron fumarolas nuevas y fuentes termales en el fondo del cráter Principal y se formó de nuevo la laguna. Estos cambios alertaron a los vulcanólogos del ICE, la UCR y la UNA sobre la posibilidad de una reactivación del volcán (Barquero et al., 1995b). A partir del 27 de octubre de 1994, se registraron nuevos enjambres sísmicos, localizados a una distancia entre 0,5 y $10 \mathrm{~km}$ del cráter Principal, varios de ellos entre 4,0 y 4,5 km de profundidad y con magnitudes entre 1,7 y 2,6 (Fernández et al., 1994; BGVN, 1994).

El OVSICORI-UNA y en parte la RSN (UCR-ICE), comunicaron que del 7 al 18 de noviembre de 1994, se produjeron 255 sismos $\left(\mathrm{M}_{\mathrm{L}}\right.$ entre 2,0 y 3,4 ), de los cuales unos 50 fueron localizados a lo largo de un segmento de falla de unos $12 \mathrm{~km}$ de longitud y orientada NW-SE y a profundidades entre $1 \mathrm{~km}$ y $29 \mathrm{~km}$ (Barquero et al., 1995a; BGVN, 1994). El OVSICORI-UNA informó que durante este período, la red de inclinómetros no detectó cambios significativos en la deformación del edificio volcánico, contrariamente a una nivelación realizada en una línea a 4 km al sur del cráter Principal, la cual mostró un pulso de inflación de $32 \mathrm{mrad}$. Sin embargo, no se reportan las incertidumbres de esta medición. Antes del 8 de diciembre y coincidiendo con la actividad sísmica, los vecinos reportaron haber escuchado "ruidos" en la pared norte y noroeste del cráter Principal del Irazú, en el área conocida como Las Fumarolas, y antiguamente denominada "Volcán Nuevo" (BGVN, 1994).

El día 8 de diciembre en la noche (10:48 p.m., hora local), en los informes se indica que hubo al menos un par de explosiones freáticas (explosiones de vapor) en el área fumarólica del flanco NW de la ladera externa al cráter Principal del volcán, que ocasionaron vibraciones en el terreno o algo similar a un sismo. La emisión de partículas fragmentadas durante estas explosiones (ceniza de materiales líticos previos, no magmáticos), se estimó que pudo haber alcanzado más $800 \mathrm{~m}$ de altura (RSN, 1994) y afectó la parte oeste-suroeste del volcán. Estas partículas fueron barridas por 


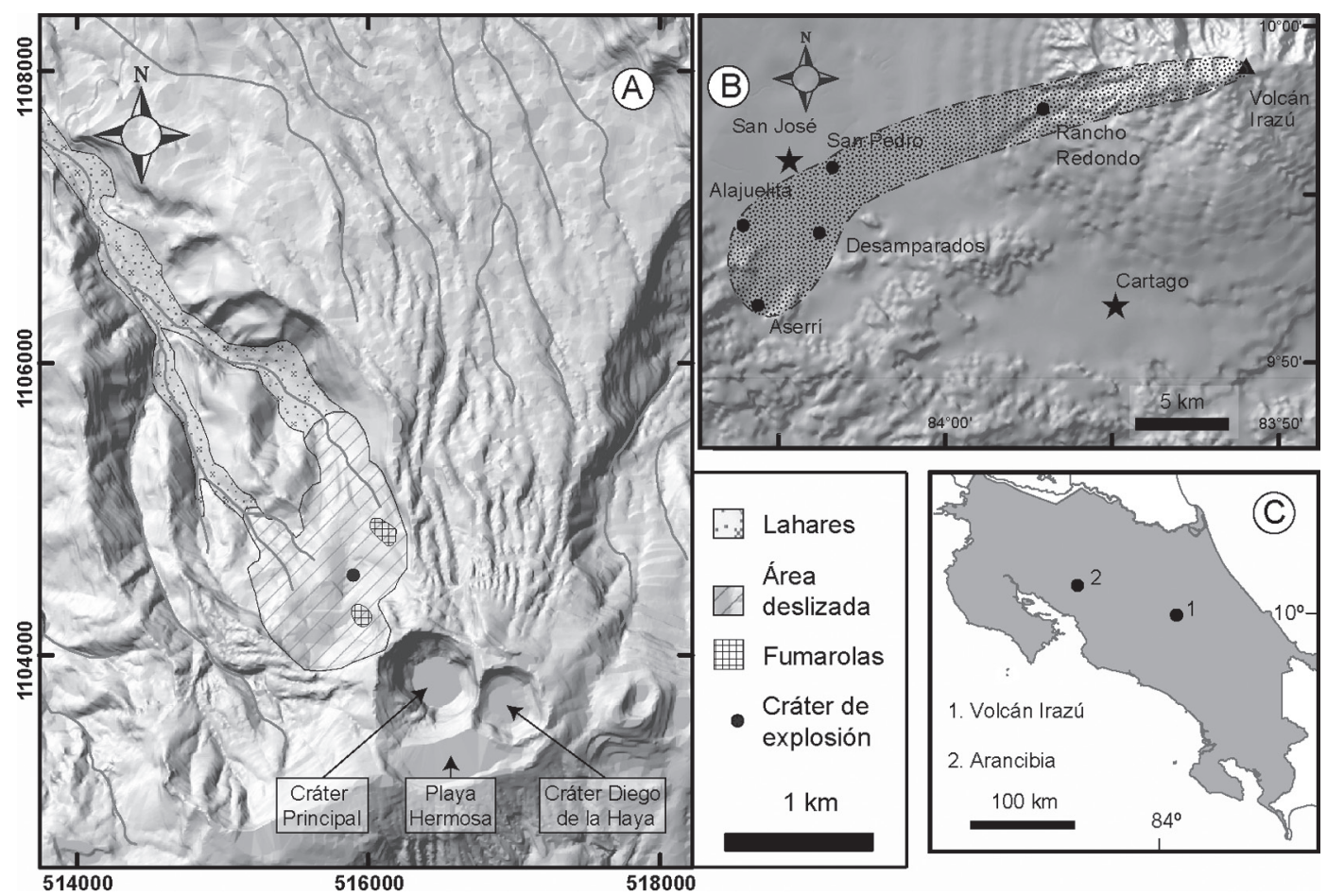

Fig. 1: Área afectada por el evento del 8 de diciembre de 1994 en el Irazú y el cráter de explosión propuesto, basado en la interpretación de Barquero et al. (1995). Inserto se presenta la ubicación del volcán Irazú (cordillera volcánica Central) y del deslizamientos de Arancibia (cordillera de Tilarán) como ejemplo comparativo, y en otro recuadro, las poblaciones en donde se registró la caída de "ceniza".

los vientos hacia el Valle Central Occidental con un azimut de $250^{\circ}$, alcanzando los poblados de Rancho Redondo, San Pedro de Montes de Oca, Desamparados, Alajuelita y Aserrí, a unos $30 \mathrm{~km}$ del volcán al WSW (BGVN, 1994). La explosión principal se describe como dirigida hacia el flanco norte, y se extendió unos 500 m, en la cabecera del río Sucio, provocando deslizamientos y lahares que se encauzaron por ese valle, particularmente a raíz de las lluvias del día siguiente, que destruyeron la vegetación varias decenas de metros sobre el nivel del río. Como consecuencia, más de $3 \mathrm{~km}^{2}$ fueron devastados por la explosión y la subsecuente erosión intensa producto de los aludes y lahares de lodo, que descendieron hasta unos 2500 m s.n.m.. Un cráter de explosión de unos 60-80 m de diámetro y deslizamientos importantes, fueron las consecuencias morfológicas más conspicuas de este evento, no relacionado directamente con el vulcanismo, sino asociado por los investigadores al sistema geotermal del volcán. Días después, se informó de la presencia de una serie de puntos de emisión de gases, con columnas de hasta $100 \mathrm{~m}$ de altura (Fernández et al, 1994; BGVN, 1994, 1995; Barquero et al., 1995; Barquero, 1998).

En los informes de la época se interpretó que las explosiones pudieron haber ocurrido debido a posibles cambios en la presión de poro en el medio rocoso, acelerado por la sismicidad y los deslizamientos en una ladera con actividad hidrotermal intensa (Fernández et al., 1994; Barquero et al., 1995a; Mora, 1997; Barquero, 1998). Sin embargo, ni en las descripciones, ni en las interpretaciones, se aclaró si las explosiones habían disparado el deslizamiento o si el deslizamiento, 
debido a la descompresión por pérdida de carga litostática, había disparado las explosiones de una "bolsa de gas hidrogeotérmica".

\section{LOS HECHOS Y LAS DUDAS}

En los documentos de la época se pueden extraer una serie de hechos que se deben de considerar como irrefutables, y que no requieren de interpretación, tal y como se puede entresacar de los trabajos de la época. Estos son:

a) Los procesos que tuvieron lugar el 8 de diciembre ocurrieron de noche y por lo tanto, nadie observó lo acontecido. Los lugareños reportaron un intenso ruido (similar al de un "jet") o vibración, algunos retumbos y presuntas explosiones.

b) Se informó acerca de la caída de ceniza hacia el occidente del Irazú, particularmente en Rancho Redondo (2040 m s.n.m.), San Pedro de Montes de Oca (1200 m s.n.m.), Desamparados (1150 m s.n.m.), Alajuelita (1020 m s.n.m.) y Aserrí (1300 m s.n.m.), a unos $30 \mathrm{~km}$ del volcán al WSW.

c) Sin ninguna duda ocurrió un gran deslizamiento y varias corrientes laháricas sobre el río Sucio.

d) Se observó en varios sectores la salida de gases con alturas de 50-100 m.

e) La actividad sísmica tuvo un pico el 9 de diciembre (unos 70 micro-temblores) y disminuyó notoriamente con una semana después del $8 \mathrm{de}$ diciembre.

Por el contrario, también hay una serie de aspectos que generan dudas e invitan a meditar y revisitar dicho evento, casi dos décadas después:

a) Ninguno de los trabajos comenta ni muestra el registro sismológico de los eventos, ni describen las señales sísmicas asociadas (frecuencias, amplitud, forma de onda, duración). Solamente se reportó que la explosión generó un sismo superficial con magnitud $M_{L} 4,4$ (RSN, 1994). Asimismo, se menciona un incremento en la actividad sísmica de alta frecuencia el 9 de diciembre, pero no se le compara con los días previos, ni se detallan las características de esa sismicidad. Por lo tanto, queda la duda si esos eventos fueron tectónicos o bien sismos de alta frecuencia pero asociados con otros procesos (p.ej. rupturas por descompresión de rocas, caída de bloques, deslizamientos). Desgraciadamente, los sismogramas no se han logrado localizar.

b) A partir de las fotografías de la época (p.ej. Barquero, 1998), se pudo constatar que todas fueron tomadas de lejos y no hay imágenes específicas del supuesto cráter de explosión. Esto sería de esperarse por lo difícil y peligroso del acceso al área. Sin embargo, de haber existido un cráter de explosión, este se debió de haber formado posterior o al final de los deslizamientos, a menos que se hubiese formado un cráter antes y otros después de los deslizamientos.

c) Para esa época, en la comunidad vulcanológica nacional existía poca experiencia en la interpretación de los depósitos de flujos de escombrosvolcánicos(volcanicdebrisavalanches), sus causas y morfología.

d) El hecho de que ocurrieran enjambres sísmicos y tremor, temporalmente cercanos, o durante el proceso, no necesariamente significa que tengan una relación directa. La ocurrencia de enjambres sísmicos y de tremor en el Irazú no es un hecho nuevo y no suele ir acompañado de deslizamientos o actividad eruptiva.

\section{ANÁLISIS FOTOGEOLÓGICO PRELIMINAR}

Algunas apreciaciones geomorfológicas preliminares se pueden concluir del análisis de las fotografías aéreas de 1978 y de 1994, así como de las imágenes tomadas por los investigadores inmediatamente después del 8 de diciembre. Aunque se espera ampliar el grado de detalle en un futuro, en este trabajo se presentan los rasgos más sobresalientes. En primera instancia, las fotografías aéreas tomadas doce días después de que ocurriera la avalancha de escombros volcánicos, se pudo constatar la magnitud del deslizamiento y sus efectos sobre la selva virgen. La altura del colapso, en ciertos sectores, fue de hasta $500 \mathrm{~m}$ (Fig. 2). Asimismo, las cicatrices de las laderas del cauce del río Sucio y de su cuenca alta, por donde circuló la avalancha, permiten deducir que el flujo tuvo que haber alcanzado una velocidad alta (quizás unos $200 \mathrm{~km} / \mathrm{h}$ ), lo que le permitió 


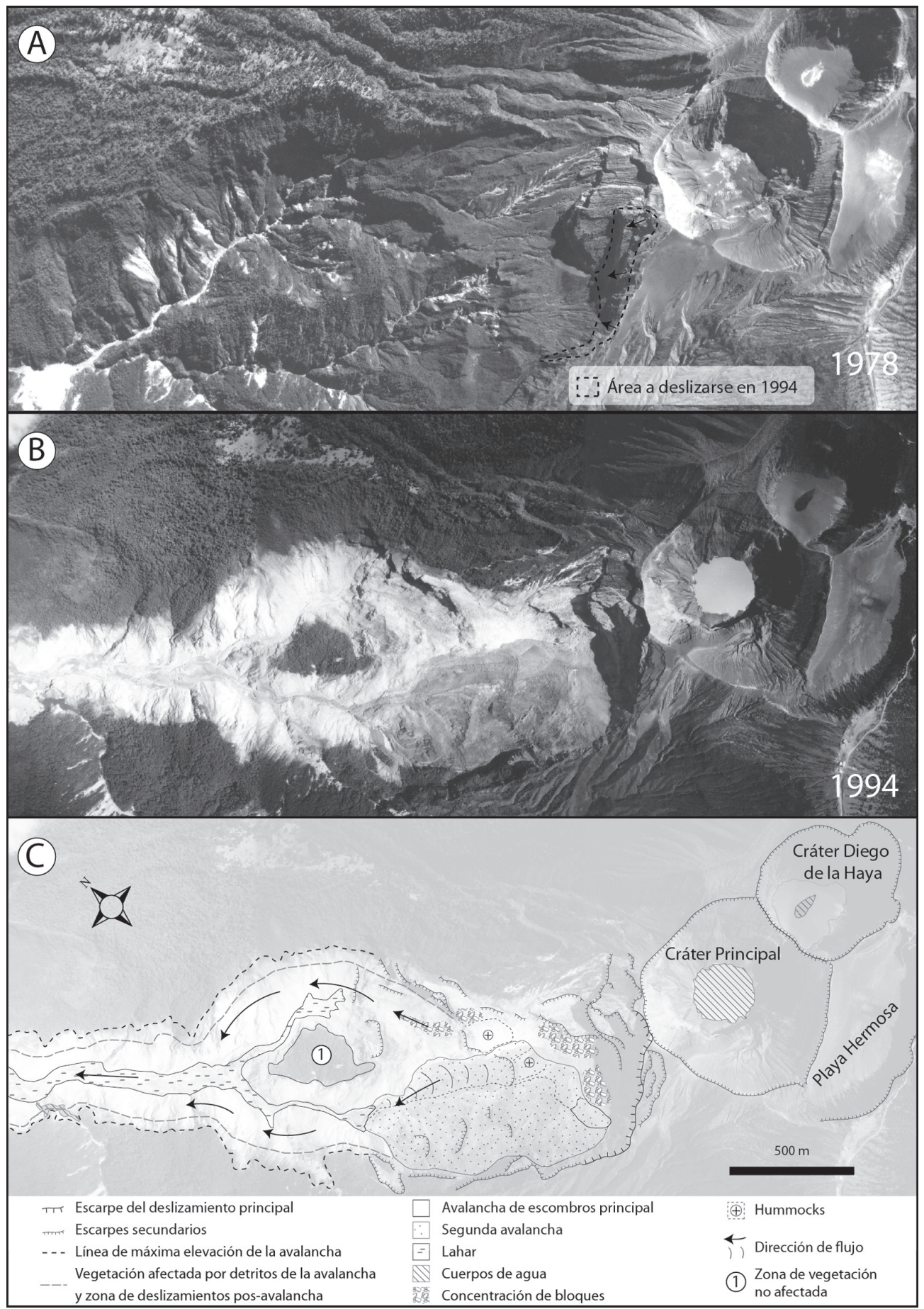

Fig. 2: Comparación del flanco norte del Irazú pre-deslizamiento con base en las fotografías aéreas de 1978 (A), y pos-avalancha con base en las fotografías aéreas del 20 de diciembre de 1994 (B). Se muestra a fotointerpretación preliminar (C) con las características morfológicas del área afectada por la avalancha, los tipos de depósitos y su secuencia relativa. Se observa que el cráter de explosión propuesto en los trabajos previos, no se visualiza en las fotografías aéreas de 1994. 
alcanzar alturas, contrapendiente, de casi $250 \mathrm{~m} \mathrm{y}$, al mismo tiempo (línea de máxima elevación de la avalancha) desestabilizar las laderas, lo que produjo deslizamientos menores a lo largo de ellas. Esta avalancha se transformó después, al incorporar agua tanto del río Sucio como de las precipitaciones, en lahares. Un hecho interesante, es que en las fotografías aéreas no se observa ningún cráter de explosión, a menos que este fuese rellenado o incluso erosionado, poco tiempo después de formado. Si se observan los sectores que colapsaron, así como dos posibles eventos principales de avalancha y varios deslizamientos menores, así como el sector de sedimentación (lahares?) en la vega del cauce. Se observan, además, posibles hummocks, representados como lomas subcónicas, que también se aprecian mejor en las fotografías de bajo ángulo tomadas por los investigadores directamente en el campo (p.ej. Barquero, 1998). De igual modo, se distinguen sectores con acumulación de bloques, que sobresalen de los depósitos circundantes.

\section{LA AVALANCHA DE ESCOMBROS DE ARANCIBIA (JUNIO DEL 2000)}

La avalancha de escombros de Arancibia, que tuvo lugar en el año 2000, presenta similitud con la del Irazú de 1994 y, por comparación, podría ilustrar y ayudar a entender lo sucedido en 1994 (Fig. 1).

El 27 de junio del 2000, aproximadamente a las 10: 30 a.m., una gran masa de rocas volcánicas y suelo se deslizó desde el cerro El Silencio (sector de Arancibia, cordillera de Tilarán). Tanto previamente como después del deslizamiento, las personas informaron de sonidos parecidos a explosiones, posiblemente debidas a la onda expansiva o a la fricción de los materiales que pudieron generar ondas sonoras, semejantes a las de algún tipo de evento sísmico de alta frecuencia. El 5 de julio se produjo un nuevo deslizamiento de menores proporciones. El primer deslizamiento generó un depósito de avalancha de escombros volcánicos (volcanic debris deposit), con todas sus características típicas, con la salvedad de que ocurrió en una región volcánicamente inactiva en la actualidad. La avalancha, durante su movimiento, levantó una columna de polvo. Casi simultáneamente, el deslizamiento se transformó en una corriente de barro debido a la incorporación del agua del río Sucio, proceso que se incrementó por las lluvias.

En el contexto anterior, las características de la avalancha de Arancibia pueden contribuir a entender su posible homóloga del Irazú en 1994. Estas características son:

a) Al ser una avalancha de escombros volcánicos, el evento de Arancibia viajó a mucha velocidad ( $\geq 113 \mathrm{~km} / \mathrm{h}$ ), e incluso se observó que la masa subió algunas laderas contrapendientes de al menos hasta $60 \mathrm{~m}$, arrasó todo a su paso, y fue claramente direccional o dirigida, como la del Irazú, lo que puede confundirse con una explosión dirigida (lateral blast deposit).

b) Tanto los deslizamientos grandes como los lahares suelen, además, generar una intensa vibración durante su movimiento (Alvarado et al., 2002, 2003; Alvarado, 2010), tal y como la referida por los vecinos del Irazú, mucho más claramente perceptible de noche, cuando el ruido ambiental y antropogénico es significativamente menor; por su intensidad, que pudo ser posteriormente interpretada como "explosiones". También se pueden presentar eventos sísmicos sonoros de alta frecuencia producida por la descompresión de las rocas, los cuales pueden interpretarse en los sismogramas como explosiones o sismos volcano-tectónicos. Aunado a ello, se sabe que los enjambres sísmicos fueron someros y que ello pudo generar ondas sonoras al transmitirse las ondas sísmicas $\mathrm{P}$ desde el suelo al aire. Adicionalmente, un sismo equivalente $M_{L} 4,4$, tal como lo refiere RSN (1994) no es raro para deslizamientos de proporciones similares. Por ejemplo, un deslizamiento menor como el del río Toro de 1992, se le asignó una magnitud equivalente de 1,43 $\mathrm{M}_{\mathrm{s}}$ y 3,4 $\mathrm{M}_{\mathrm{b}}$ (Mora et al., 1993) o bien, el colapso de las torres gemelas (EE.UU) respondieron a una $\mathrm{M}_{\mathrm{L}}$ equivalente 2,1 y 2,3 (http://www.lis.ucr.ac.cr/index.php?ib=296).

c) Más sobresaliente aún, fue el hecho de que la fragmentación de las rocas, durante el megadeslizamiento de Arancibia, generó una columna de "cenizas" que alcanzó gran altura (quizás 
unos $300 \mathrm{~m}$ ), por lo que Alvarado et al. (2003) la llaman "co-avalanche dust-cloud" (nube de polvo co-avalancha). Este resulta ser un caso muy interesante de auto-fragmentación, poco descrito en la literatura, aunque no excepcional. Al polvo resultante no se le debe llamar "ceniza", dado que la fragmentación no es el producto de un proceso explosivo originado por la desgasificación. Lo anterior motivó que los lugareños pensaran que en la localidad de Arancibia estaba "...naciendo un volcán...", y a la existencia de un olor sulfurado, producto de la alteración hidrotermal del tipo epitermal. Ello podría, también, explicar la formación de "cenizas" en el Irazú (que posiblemente alcanzaron elevaciones de hasta unos $3700 \mathrm{~m}$ s.n.m.) y su caída hacia el occidente, llevada por los vientos alisios, hasta lugares a alturas inferiores entre los 2000 y 1000 m s.n.m. (Figs. 1 y 3).

d) El hecho de que el evento del 8 de diciembre ocurriera en el flanco norte del Irazú, muy cerca de las fumarolas, en un volcán históricamente activo y asociado espacio-temporalmente con la sismicidad, pudo haber influido en los investigadores de ese entonces, para concluir que se tratara de una explosión freática. El evento de Arancibia ocurrió en plena estación lluviosa y, sin embargo, las rocas generaron polvo, porque claramente no estaban saturadas todas ellas (tan solo localmente los espacios comunicantes y a nivel de los acuíferos locales), por lo que no sería extraño la generación de polvo al final de la época lluviosa, en el caso de una avalancha rocosa, como fue el caso del Irazú en 1994.

\section{DISCUSIÓN}

Es claro que el 8 de diciembre ocurrieron al menos dos deslizamientos grandes en el flanco norte del Irazú, en un sector con numerosas fallas y con laderas inestables, en un terreno con pendiente empinada, alteración hidrotermal, buzamiento a favor de pendiente de las capas volcánicas y elevada precipitación pluvial. Los depósitos se transformaron en lahares al incorporar las aguas del río Sucio. Al analizarse las fotografías tomadas por diversos investigadores en el sector norte del Irazú, se puede concluir
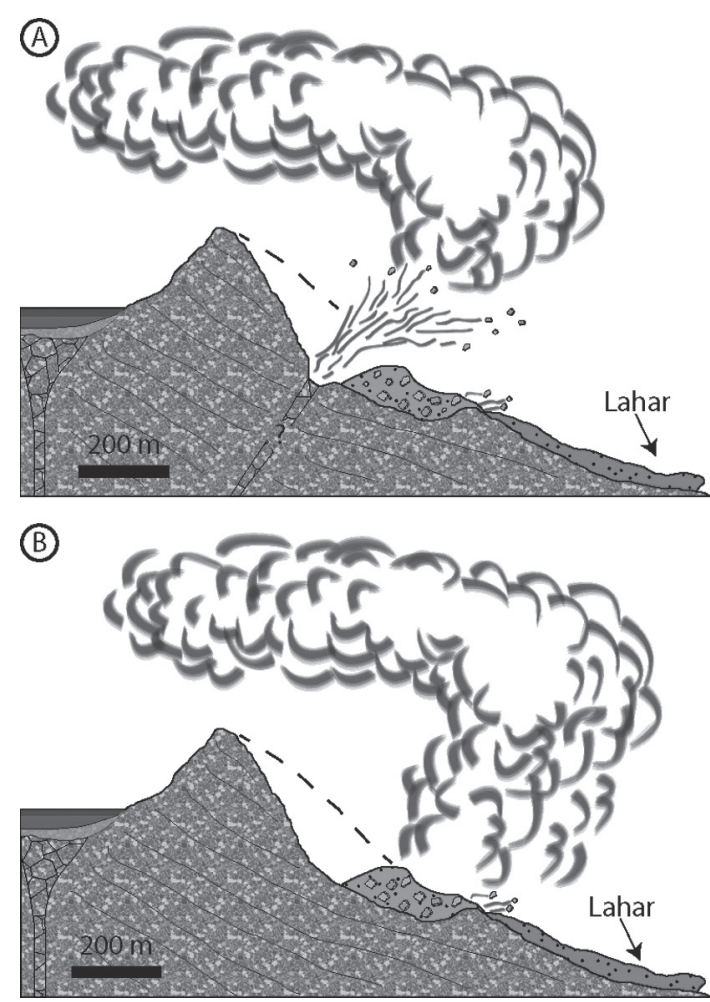

Fig. 3: Los dos interpretaciones posibles para el evento del Irazú del 8 de diciembre de 1994: A) Deslizamiento y explosión freática dirigida, con penacho de cenizas y generación de lahares a lo largo del cauce del río Sucio. El deslizamiento pudo disparar la explosión o viceversa, la explosión, de haberse producido, también pudo disparar el deslizamiento. B) Deslizamiento tipo avalancha de escombros rocosos, con nube de polvo acompañante debido a la auto-fragmentación. En ambos modelos, la generación de los lahares hacia aguas abajo del río Sucio, se produjo por la incorporación de agua en la avalancha procedente del río y por la precipitación pluvial elevada durante esas fechas.

que pudo tratarse de un evento de avalancha de escombros volcánicos con morfología hummocky, tal y como se han descrito en otros sectores del volcán (Alvarado et al., 2004). El supuesto cráter de explosión, aunque pobremente descrito, se menciona como pequeño (60-80 m de diámetro, profundidad no establecida) e irregular, por lo perfectamente podría corresponder con una depresión típica de los depósitos de avalanchas de escombros. Las fotografías aéreas tomadas el 20 de diciembre de 1994, no dejan entrever dicho cráter (Fig. 2). 
Aunque hubo un incremento el día posterior al 8 de diciembre de la actividad sísmica (eventos de alta y baja frecuencia, así como tremor), se describe que ésta decreció rápidamente en los siguientes cinco días. Sin embargo, ello puede relacionarse tanto con un evento de explosión freática, como con eventos de descompresión de rocas, en un macizo con actividad geotermal, y con deslizamientos asociados, que pueden generar eventos sísmicos de corta duración pero de alta frecuencia, confundibles por ello con temblores volcano-tectónicos; la vigorosa desgasificación de las fumarolas, pudo generar sismos de baja frecuencia, incluyendo los tremores.

La caída de polvo al occidente del Irazú, por su parte, más que un evento explosivo, se podría interpretar, de manera alterna, como una nube de polvo acompañante a la avalancha, producto de la auto-fragmentación de los materiales rocosos, nube que fue transportada por los vientos alisios a alturas relativamente bajas. La existencia de una explosión freática, por consiguiente, no queda demostrada y, aunque no se puede descartar, pareciera que hay opciones más plausibles.

\section{CONCLUSIÓN}

El evento del 8 de diciembre resulta ser un caso interesante para el análisis, en donde se dieron por sentadas ciertas conclusiones, debido a la predisposición de concluir con base en el contexto geológico (volcán recientemente activo) y la cadena de eventos (sismicidad - ruido fuerte - caída de "cenizas" - deslizamiento - lahares). Dicho suceso, alternativamente, se podría re-interpretar como una avalancha de escombros volcánicos con auto-fragmentación y la subsiguiente generación de polvo en el sentido estricto y no de "cenizas" no juveniles. Lo anterior puede también puede utilizarse como modelo para otros eventos similares sucedidos en otras regiones o como base para casos futuros en terrenos volcánicos activos.

\section{AGRADECIMIENTOS}

Al Instituto Costarricense de Electricidad y la Universidad de Costa Rica por todo el apoyo brindado por más de dos décadas. Un revisor anónimo y en particular al Dr. Sergio Mora por sus atinados comentarios, que mejoraron mucho el presente trabajo. Dicha investigación forma parte del Estudio de deformación en volcanes activos de Costa Rica Nº113-B1-230. El Geógrafo Giovanni Oconitrillo, del Instituto Geográfico Nacional (IGN), suministró la información para localizar las fotografías aéreas tomadas después de la avalancha por el IGN en diciembre 1994.

\section{REFERENCIAS}

ALVARADO, G.E., 1987: El volcán Irazú: Síntesis geológica, actividad eruptiva y peligro volcánico preliminar.- 46 págs. Departamento de Geología, Inst. Costarricense de Electricidad, San José [Inf. Interno].

ALVARADO, G.E., 1993: Volcanology and Petrology of Irazú Volcano, Costa Rica.$\mathrm{xxv}+261$ págs. Univ. Kiel, Alemania [Tesis Ph.D.].

ALVARADO, G.E., 2010: Aspectos geohidrológicos y sedimentológicos de los flujos de lodo asociados al terremoto de Cinchona $\left(\mathrm{M}_{\mathrm{w}}\right.$ 6,2) del 8 de enero del 2009, Costa Rica.- Rev. Geól. Amér. Central, 43: 67-96. 
ALVARADO, G.E., 2011: Los volcanes de Costa Rica: Geología, historia, riqueza natural y su gente.- 335 págs. EUNED, San José.

ALVARADO, G.E., PÉREZ, W. \& SIGARÁN, C., 2000: Vigilancia y peligro volcánico.- En: DENYER, P. \& KUSSMAUL, S. (eds.): Geología de Costa Rica.- Ed. Tecnológica de Costa Rica, Cartago: 251-272.

ALVARADO, G.E., ARCE, R., SÁNCHEZ, J.M., 2002: La Caída de Grandes Bloques y la Generación de Debris Flows y su peligro implícito en los sectores residenciales de Escazú y Santa Ana.- VIII Seminario Nacional de Geotecnia, $3^{\text {er }}$ Encuentro Centroamericano de Geotecnistas: "Geotecnia en la Prevención de Desastres en el Entorno". San José: 77-82.

ALVARADO, G.E., MORA, R. \& PERALDO, G., 2003: The June 2000 Arancibia Debris Avalanche and Block-slide, Costa Rica.Landslide News, 14/15: 29-32.

ALVARADO, G.E., VEGA, E., CHAVES, J. \& VÁZQUEZ, M., 2004: Los grandes deslizamientos (volcánicos y no volcánicos) de tipo debrisavalanche en Costa Rica.- Rev. Geol. Amér. Central, 30: 83-99.

BARQUERO, J., 1998: Volcán Irazú, Costa Rica.- 49 págs. Impreso Lihssa, San José.

BARQUERO, R., MORA, M., MADRIGAL, L., VARGAS, I., ARIAS, F. \& SOTO, G., 1995a: Resumen anual de sismos y actividad volcánica en Costa Rica durante 1994.- 19 págs. Oficina de Sismología y Vulcanología, RSN [Inf. Interno].
BARQUERO, R., LESAGE, P., METAXIAN, J.P., CREUSOT, A. \& FERNÁNDEZ, M., 1995b: La crisis sísmica en el volcán Irazú en 1991 (Costa Rica).- Rev. Geol. Amér. Central, 18: 5-18.

BGVN, 1994: Irazú Monthly Reports.Global Volcanism Program. http:www. volcano.si.edu/would/volcano. $\mathrm{cfm}$ ? vnum $=1405-06=\&$ volpage $=$ var, 10-12/1994 [Consulta: 2 de diciembre de 2013].

BGVN, 1995: Irazú Monthly Reports.- Global Volcanism Program. http:www.volcano. si.edu/would/volcano.cfm?vnum=140506=\&volpage $=$ var,01/1995 [Consulta: 26 de febrero de 2013].

FERNÁNDEZ, E., BARQUERO, J., BARBOZA, V., VAN DER LAAT, R., MALAVASSI, E., SÁENZ, R., MARINO, T. \& MARTÍNEZ, M., 1994. Estado de los volcanes en 1994.Bol. Vulcanol. OVSICORI, http://www. ovsicori.una.ac.cr/index.php) [Consulta: 14 de febrero de 2013].

MORA, M., 1997: Informe sobre la actividad de los volcanes Poás e Irazú 1994-1996.- 64 págs. Red Sismológica Nacional (RSN: ICE-UCR), San José [Inf. Interno].

MORA, M., ASH, C., OCONITRILlO, G. \& SALAZAR, L.G., 1996: Amenaza y vulnerabilidad en el flanco norte del Volcán Irazú: Explosión de diciembre de 1994, un caso específico. IV Simposio Latinoamericano sobre Riesgos Geológicos en Áreas Urbanas. Universidad de Costa Rica, San José: 58. 
RSN, 1994: Boletín Sismológico y Vulcanológico, Diciembre 1994.- 6 págs. Univ. Costa Rica, Instituto Costarricense de Electricidad, San José [Inf. Interno].

RSN, 1995: Resumen de actividad sísmica y volcánica. Periodo de Enero - Diciembre de 1994.- Rev. Geol. Amér. Central, 18: 97-100.
MORA, S., MADRIGAL, C., ESTARADA, J. \& SCHUSTER, R.L., 1993. The 1992 Rio Toro Landslide Dam, Costa Rica.Landslide News, 7: 19-22.

SIEBERT, L., SIMKIN, T. \& KIMBERLY, P., 2010: Volcanoes of the World.- 551 págs. Smithsonian Inst.- Univ. California Press, Berkeley and Los Angeles, California. 